United Nations Educational Scientific and Cultural Organization and

International Atomic Energy Agency

THE ABDUS SALAM INTERNATIONAL CENTRE FOR THEORETICAL PHYSICS

\title{
PROJECTIVE NORMALITY OF THE WONDERFUL COMPACTIFICATION OF SEMISIMPLE ADJOINT GROUPS
}

\author{
S. Senthamarai Kannan ${ }^{1}$ \\ Chennai Mathematical Institute, 92, GN Chetty Road, Chennai-17, India \\ and \\ The Abdus Salam International Centre for Theoretical Physics, Trieste, Italy.
}

\begin{abstract}
We prove that if $G$ is a semisimple adjoint group over an algebraically closed field of arbitrary characteristic $\Gamma X$ is the wonderful compactification of $G$ and $\widehat{G}$ is a simply connected covering of $G \Gamma$ then for any ample line bundle $L$ over $X \Gamma$ the cone over $X$ given by $L$ is normal.
\end{abstract}

MIRAMARE - TRIESTE

October 2000

${ }^{1}$ E-mail: kannan@ictp.trieste.it; kannan@smi.ernet.in 


\section{Introduction}

In [4] $\Gamma$ what are known as "wonderful compactifications" of symmetric varieties were constructed and studied by C. De Concini and C. Procesi. More preciselyГif $G$ is a semisimple adjoint group over the field of complex numbers $\Gamma H$ is the subgroup of all fixed points of an involution $\sigma$ of $G$ that is induced by an involution $\widehat{\sigma}$ of the simply connected covering $\widehat{G}$ of $G \Gamma$ then $\Gamma$ they have constructed a complete embedding $\overline{G / H}$ of the homogeneous space $G / H$ Twith boundary being a union of normal crossing divisors. In particularГone gets such a compactification $\bar{G}$ for the group $G$ ( $G$ being considered as $(G \times G) / \Delta(G))$. In [10] $\Gamma$ E.Strickland has proved that the wonderful compactifications for the group exists over algebraically closed fields of positive characteristics. In [5] ГC. De Concini and T.A. Springer have proved that these compactifications for arbitrary symmetric space $G / H$ exists when the base field is of characteristic $p \neq 2$. In $[6] \Gamma \mathrm{G}$. Faltings raised the question: For what $\widehat{G}$ - linearised line bundles $L \Gamma$ the cone over $\overline{G / H}$ given by $L$ is normal?

The aim of this paper is to provide an affirmative answer to his question for the case of the wonderful compactification of a semisimple adjoint group $G$ over an algebraically closed field of arbitrary characteristic. To be more precise $\Gamma$ we prove that if $G$ is a semisimple adjoint group over an algebraically closed field of arbitrary characteritic $\Gamma \widehat{G}$ is a simply connected covering of $G \Gamma$ then for any $\widehat{G} \times \widehat{G}$ - linearised very ample line bundle $L$ over the woderful compactification $\bar{G} \Gamma$ the cone over $\bar{G}$ given by $L$ is normal. In section $3 \Gamma$ we prove this result when the base field is the field of complex numbers. For a more precise statement $\Gamma$ see Theorem 3.4. In section $4 \Gamma$ we prove the result for algebraically closed fields of positive characteristic by using the properties of good filtrations.

\section{Notations and basic Theorems}

Throughout sections 2 and 3, we fix the following notations: Let $G$ be a semisimple adjoint group over the field of complex numbers. Let $\pi: \widehat{G} \longrightarrow G$ be a simply connected covering of $G$. Let $k[\widehat{G}]$ denote the co-ordinate ring of $\widehat{G}$. Let $T$ be a maximal torus of $G$ and $B$ be a Borel subgroup of $G$ containing $T$. Let $B^{-}$be the opposite Borel subgroup of $G$ determined by $T$ and $B$. Let $\widehat{T}$ denote the pull back of $T$ in $\widehat{G}$. Let $\widehat{B}$ (resp. $\widehat{B^{-}}$) denote the pull back of $B$ (resp. $B^{-}$) in $\widehat{G}$. Let $W$ denote the Weyl group of $G$ with respect to $T$.

Let $\Phi$ denote the set of roots with respect to $T$ as above and $\Phi^{+} \subset \Phi$ denote the set of positive roots with respect to $B$ as above. 
Let $\Delta \subset \Phi^{+}$denote the set of simple roots. We label the elements of $\Delta$ by :

$$
\Delta:=\left\{\alpha_{1}, \alpha_{2}, \ldots \alpha_{l}\right\}, l=\operatorname{rank}(G)
$$

Let $\chi$ denote the weight lattice of $G$ with respect to $T$. Let $E:=\chi \otimes \mathbb{R}$ denote the $\mathbb{R}$ span of $\chi$. Let $(.,$.$) denote the positive definite bilinear form on E$ induced by the Killing form of the Lie algebra of $G$ and define $\langle\alpha, \beta\rangle=\frac{2(\alpha, \beta)}{(\beta, \beta)}$. Now $\Gamma$ consider the fundamental weights. Since $\Gamma$ they form a dual basis of the simple coroots $\Gamma$ we label them:

$$
\left\{\varpi_{1}, \ldots \varpi_{l}\right\}
$$

where $\varpi_{i}$ is dual to $\alpha_{i}$. Let $w_{0}$ denote the longest element of $W$ with respect to $\Delta$.

In this paragraph $\Gamma$ we recall some basic properties of the wonderful compactification of the symmetric spaces. Let $G$ be a semisimple adjoint group $\Gamma$ let $\sigma$ be an involution of $G$ that is induced by an involution $\widehat{\sigma}$ of $\widehat{G}$. Let $H$ denote the subgroup of all fixed points of $\sigma$ in $G$. Let $H^{\prime}$ denote the fixed points of $\widehat{\sigma}$ in $\widehat{G}$. Then $\Gamma$ there exist a $\sigma$-stable maximal torus $T$ of $G$ and a Borel subgroup $B$ of $G$ containing $T$ with the following property :

1. The dimension of $\left\{t \in T: \sigma(t)=t^{-1}\right\}$ is maximal.

2. For any positive root $\alpha$ (with respect to $B$ ) $\Gamma$ if $\sigma(\alpha)$ is a positive $\operatorname{root} \Gamma \operatorname{then} \sigma(\alpha)=\alpha$.

Let $\left\{\gamma_{1}, \gamma_{2}, \cdots \gamma_{j}\right\}$ be the set of all simple roots that are not fixed by $\sigma$. Then $\sigma \sigma$ induces a permutation $\widetilde{\sigma}$ on the set $\{1,2, \cdots j\}$.

Also $\Gamma$ there is only one closed $G$-orbit in the wonderful compactification $\overline{G / H}$ एsay $G / P$ and the restriction map $\operatorname{Pic}(\overline{G / H}) \longrightarrow \operatorname{Pic}(G / P)$ is injective. For a dominant weight $\lambda \Gamma$ let $V_{\lambda}$ denote the irreducible representation of $\widehat{G}$ with highest weight $\lambda$. Let $\lambda$ be a dominant weight such that $V_{\lambda}^{H^{\prime}}$ is non zero. Then $\Gamma[$ by Theorem $8.3 \Gamma \mathrm{pp} 30-31$ [4]] $\Gamma$ the global sections of the line bundle $L_{\lambda}$ on $\overline{G / H}$ is given by:

$2.1 H^{0}\left(\overline{G / H}, L_{\lambda}\right)=\bigoplus_{\nu \in \Gamma_{\lambda}} V_{\nu}^{*} \Gamma$ where $\Gamma_{\lambda}$ denote the set of all dominant weights $\nu$ that are of the form $\nu=\lambda-\left(\sum_{i \leq \widetilde{\sigma}(i)} m_{i}\left(\gamma_{i}-\sigma\left(\gamma_{i}\right)\right)\right) \Gamma$ with $m_{i} \in \mathbb{Z}_{\geq 0}$.

Now $\Gamma$ we apply these to our special involution $\sigma: G \times G \longrightarrow G \times G$ sending $(x, y) \mapsto(y, x)$.

Here $\Gamma$ the symmetric space is the group $G=(G \times G) / \Delta(G)$. Let $X$ denote the wonderful compactification of $G$.

Here $\Gamma$ we should take $T \times T$ as a $\sigma$ - stable maximal torus with the above maximal property. We should take $B \times B^{-}(T \subset B)$ as a Borel subgroup of $G \times G$ containing $T \times T$ with the above property. Here $\Gamma$ the unique closed $G \times G$ - orbit is $G / B \times G / B^{-} \Gamma$ and the image of $\operatorname{Pic}(X) \longrightarrow \operatorname{Pic}\left(G / B \times G / B^{-}\right)$consists of line bundles associated to weights of the form $(\lambda,-\lambda)$. For any dominant weight $\lambda \Gamma$ let $L_{\lambda}$ denote the line bundle over $X$ associated to $\lambda$ and let 
$H^{0}\left(X, L_{\lambda}\right)$ denote the global sections of $L_{\lambda}$ over $X$. Let $\leq$ denote the dominant ordering on $\chi$. That is $\nu \leq \lambda$ if and only if $\lambda-\nu=\sum_{i=1} m_{i} \alpha_{i}$ for some $m_{i} \in \mathbb{Z}_{\geq 0}$.

Now Tapplying the above statement 2.1 to our special involution of $G \times G$ Twe have $2.2 H^{0}\left(X, L_{\lambda}\right)=\bigoplus_{\nu \leq \lambda} \operatorname{End}\left(V_{\nu}^{*}\right) \Gamma$ where the sum is taken over all dominant weights $\nu$ such that $\nu \leq \lambda$.

\section{Projective normality}

We now prove that for any line bundle $L_{\lambda}$ on $X \Gamma$ there is a unique (up to multiplication by a constant) injective homomorphism

$$
\phi_{\lambda}: H^{0}\left(X, L_{\lambda}\right) \rightarrow k[\widehat{G}]
$$

of $\widehat{G} \times \widehat{G}$ modules.

For a proof of this fact: Since $G=G \times G / \Delta(G)$ is a $G \times G$ stable open subset of $X$ Tand the natural map $\pi: \widehat{G}=\widehat{G} \times \widehat{G} / \Delta(\widehat{G}) \longrightarrow G=G \times G / \Delta(G)$ is a surjection $\Gamma$ the natural map $\pi: \widehat{G}=\widehat{G} \times \widehat{G} / \Delta(\widehat{G}) \longrightarrow X$ is a dominant map and hence for any line bundle $L$ over $X \Gamma H^{0}(X, L)$ is a $\widehat{G} \times \widehat{G}$ - sub module of the $\widehat{G} \times \widehat{G}$ module $H^{0}(\widehat{G}, L)$. Since $k[\widehat{G}]$ is a unique factorisation domain $\Gamma$ the pull back $\pi^{*}(L)$ on $\widehat{G}$ is trivial and hence we have

$$
H^{0}(\widehat{G}, L)=k[\widehat{G}]
$$

Moreover $\Gamma$ this identification is unique up to constants since the units of the $k$-algebra $k[\widehat{G}]$ are only nonzero constants. Thus $\Gamma(3.0 .1)$ holds.

We now prove that for any two line bundles $L_{\lambda}$ and $L_{\mu}$ Tthe following diagram is commutative:

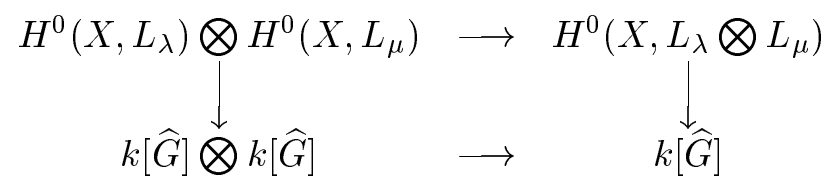

Here the horizontal arrows are the natural maps $\Gamma$ the vertical map on the left is $\phi_{\lambda} \otimes \phi_{\mu}$ and the vertical map on the right is $\phi_{\lambda+\mu}$.

For a proof of this observation:

By the Peter-Weyl Theorem $\Gamma$ the $\widehat{G} \times \widehat{G}$ - module $k[\widehat{G}]=\bigoplus_{\nu} \operatorname{End}\left(V_{\nu}^{*}\right) \Gamma$ where the sum is taken over all dominant weights $\nu$. Using this and the fact that $H^{0}\left(X, L_{\lambda}\right)=\bigoplus_{\nu \leq \lambda} \operatorname{End}\left(V_{\nu}^{*}\right) \Gamma$ it is easy to see that (3.0.2) holds.

Now $\Gamma$ we prove the following Lemma. 
Lemma 3.1. Let $\lambda$ and $\mu$ be two dominant weights. Let $\lambda_{1}$ be a dominant weight such that $\lambda_{1} \leq \lambda$ and $\mu_{1}$ be a dominant weight such that $\mu_{1} \leq \mu$. Let $\nu$ be a dominant weight such that $V_{\nu}$ is a $\widehat{G}$ - submodule of the tensor product $V_{\lambda_{1}} \otimes V_{\mu_{1}}$. Then, the image of $\operatorname{End}\left(V_{\lambda_{1}}^{*}\right) \otimes \operatorname{End}\left(V_{\mu_{1}}^{*}\right)$ under the multiplication map $H^{0}\left(X, L_{\lambda}\right) \otimes H^{0}\left(X, L_{\mu}\right) \longrightarrow H^{0}\left(X, L_{\lambda+\mu}\right)$ contains End $\left(V_{\nu}^{*}\right)$.

Proof: Since $\widehat{G}$ is linearly reductive and $V_{\nu}^{*}$ is a quotient of $V_{\lambda_{1}}^{*} \otimes V_{\mu_{1}}^{*}$ Twe can assume that $V_{\nu}^{*}$ is a submodule of $V_{\lambda_{1}}^{*} \otimes V_{\mu_{1}}^{*}$.

Now $\Gamma$ consider the $\widehat{B}$ action on $\operatorname{End}\left(V_{\lambda_{1}}^{*}\right)$ on the right and consider the $\widehat{B^{-}}$action on $\operatorname{End}\left(V_{\mu_{1}}^{*}\right)$ on the right. By the observation 2.2 and the hypothesis $\lambda_{1} \leq \lambda$ and $\mu_{1} \leq \mu$ एwe have $\operatorname{End}\left(V_{\lambda_{1}}^{*}\right) \subset$ $H^{0}\left(X, L_{\lambda}\right)$ and $\operatorname{End}\left(V_{\mu_{1}}^{*}\right) \subset H^{0}\left(X, L_{\mu}\right)$. So $\Gamma$ by our identification (3.0.1) $\Gamma$ we have $\operatorname{End}\left(V_{\lambda_{1}}^{*}\right) \subset$ $H^{0}\left(X, L_{\lambda}\right) \subset k[\widehat{G}]$ and $\operatorname{End}\left(V_{\mu_{1}}^{*}\right) \subset H^{0}\left(X, L_{\mu}\right) \subset k[\widehat{G}]$. Therefore [1]] Twe have $V_{\lambda_{1}}^{*}=\left\{f \in \operatorname{End}\left(V_{\lambda_{1}}^{*}\right): f(x b)=\lambda_{1}(b) . f(x)\right.$ for $\left.x \in \widehat{G}, b \in \widehat{B}\right\}=\{f \in k[\widehat{G}]: f(x b)=$ $\lambda_{1}(b) . f(x)$ for $\left.x \in \widehat{G}, b \in \widehat{B}\right\}$ and $V_{\mu_{1}}^{*}=\left\{f \in \operatorname{End}\left(V_{\mu_{1}}^{*}\right): f\left(x b^{-}\right)=w_{0}\left(\mu_{1}\right)\left(b^{-}\right) . f(x)\right.$ for $x \in$ $\left.\widehat{G}, b^{-} \in \widehat{B^{-}}\right\}=\left\{f \in k[\widehat{G}]: f\left(x b^{-}\right)=w_{0}\left(\mu_{1}\right)\left(b^{-}\right) . f(x)\right.$ for $\left.x \in \widehat{G}, b^{-} \in \widehat{B^{-}}\right\}$.

Fix our choice of $V_{\lambda_{1}}^{*}$ and $V_{\mu_{1}}^{*}$ as above and let $f=\sum_{i} f_{i} \otimes g_{i} \in V_{\lambda_{1}}^{*} \otimes V_{\mu_{1}}^{*} \Gamma f_{i} \in V_{\lambda_{1}}^{*} \Gamma$ $g_{i} \in V_{\mu_{1}}^{*}$ be such that $\left(\sum_{i} f_{i} \otimes g_{i}\right)(x, x)$ is zero $\Gamma$ for every $x \in \widehat{G}$. Then $\Gamma$ we have $f\left(\left(x b, x b^{-}\right)\right)=$ $\left(\lambda_{1}(b)\right)\left(w_{0}\left(\mu_{1}\right)\left(b^{-}\right)\right) f(x, x)=0$ for every $x \in \widehat{G} \Gamma b \in B$ and $b^{-} \in B^{-}$. Since the elements $\left(x b, x b^{-}\right)$form a dense open set in $\widehat{G} \times \widehat{G} \Gamma f$ must vanish identically on $\widehat{G} \times \widehat{G}$. Thus $\Gamma V_{\lambda_{1}}^{*} \otimes V_{\mu_{1}}^{*}$ is mapped injectively via the multiplication map $\operatorname{End}\left(V_{\lambda_{1}}^{*}\right) \otimes \operatorname{End}\left(V_{\mu_{1}}^{*}\right) \subset k[\widehat{G}] \otimes k[\widehat{G}] \longrightarrow k[\widehat{G}]$. By the hypothesis $\lambda_{1} \leq \lambda \Gamma \mu_{1} \leq \mu$ and by Theorem(2.1) $\Gamma$ we have $\operatorname{End}\left(V_{\lambda_{1}}^{*}\right) \subset H^{0}\left(X, L_{\lambda}\right)$ and $\operatorname{End}\left(V_{\mu_{1}}^{*}\right) \subset H^{0}\left(X, L_{\mu}\right)$. Therefore $\Gamma$ by the observation (3.0.2) $\Gamma$ the $\widehat{G}$ module $V_{\lambda_{1}}^{*} \otimes V_{\mu_{1}}^{*}$ is mapped injectively into $H^{0}\left(X, L_{\lambda+\mu}\right)$ via the multiplication map $H^{0}\left(X, L_{\lambda}\right) \otimes H^{0}\left(X, L_{\mu}\right)$ $\longrightarrow H^{0}\left(X, L_{\lambda+\mu}\right)$. Since $V_{\nu}^{*}$ is a $\widehat{G}$ - submodule of $V_{\lambda_{1}}^{*} \otimes V_{\mu_{1}}^{*} \Gamma V_{\nu}^{*}$ must be isomorphic to a $\widehat{G}$ submodule of the image of $\operatorname{End}\left(V_{\lambda_{1}}^{*}\right) \otimes \operatorname{End}\left(V_{\mu_{1}}^{*}\right)$. Since the image of $\operatorname{End}\left(V_{\lambda_{1}}^{*}\right) \otimes \operatorname{End}\left(V_{\mu_{1}}^{*}\right)$ is a $\widehat{G} \times \widehat{G}$ submodule of $k[\widehat{G}] \Gamma$ it must contain $\operatorname{End}\left(V_{\nu}^{*}\right)$.

Therefore $\Gamma$ using the diagram (3.0.2) $\Gamma$ it is easy to see that $\operatorname{End}\left(V_{\nu}^{*}\right)$ is a $\widehat{G} \times \widehat{G}$-submodule of the image of $H^{0}\left(X, L_{\lambda}\right) \otimes H^{0}\left(X, L_{\mu}\right) \rightarrow H^{0}\left(X, L_{\lambda+\mu}\right)$ under the multiplication map.

This completes the proof of the Lemma.

We now prove the following Lemma.

Lemma 3.2. Let $\lambda$ and $\mu$ be two dominant weights. Let $\nu$ be a dominant weight satisfying $\nu \leq \lambda+\mu$. Then, there is a dominant weight $\lambda_{1} \leq \lambda$ and there is a dominant weight $\mu_{1} \leq \mu$ such that $V_{\nu}$ is $\widehat{G}$ - submodule of the tensor product $V_{\lambda_{1}} \otimes V_{\mu_{1}}$.

Proof: Let $\lambda$ and $\mu$ be two dominant weights. Let $\nu$ be a dominant weight such that $\nu \leq \lambda+\mu$. We wish to prove that there are dominant weights $\lambda_{1} \Gamma \mu_{1}$ such that $\lambda_{1} \leq \lambda \Gamma \mu_{1} \leq \mu$ 
and an element $w$ of the Weyl group $W$ such that $\nu=\lambda_{1}+w\left(\mu_{1}\right)$ Tand then using PRV conjecture [cf [9]] $\Gamma$ we prove that $V_{\nu}$ is a $\widehat{G}$ - submodule of the tensor product $V_{\lambda_{1}} \otimes V_{\mu_{1}}$.

By [sections (13.4) and (21.3)] of [8]] $\nu$ is a weight of $V_{\lambda+\mu}$. Therefore $\Gamma$ we have $\nu=\nu_{1}+\nu_{2} \Gamma$ where $\nu_{1}$ is a weight of $V_{\lambda}$ and $\nu_{2}$ is a weight of $V_{\mu}$. We have $\lambda-\nu_{1}=\left(\sum_{i}^{l} m_{i} \alpha_{i}\right)$ for some $m_{i} \in \mathbb{Z}_{\geq 0}$.

We first prove that there is a dominant weight $\lambda_{1}$ such that $\lambda_{1} \leq \lambda$ and there is a weight $\nu_{2}^{\prime}$ of $V_{\mu}$ such that $\nu=\lambda_{1}+\nu_{2}^{\prime}$ by induction on the sum $\sum_{i}^{l} m_{i}$.

If $\sum_{i}^{l} m_{i}=0 \Gamma$ there is nothing to prove.

If $\sum_{i}^{l} m_{i}=1 \Gamma$ then $\Gamma$ we have $\nu_{1}=\lambda-\alpha_{i_{0}}$ for some $i_{0} \in\{1,2, \cdots l\}$. Now $\Gamma$ if $\nu_{1}$ is dominant then there is nothing to prove. Otherwise $\Gamma\left\langle\nu_{1}, \alpha_{i_{0}}\right\rangle$ is negative. Since $\nu=\nu_{1}+\nu_{2}$ is dominant $\Gamma$ $\left\langle\nu_{2}, \alpha_{i_{0}}\right\rangle$ is positive. But $\Gamma$ we know that $\nu_{1}+k \alpha_{i_{0}}$ is a weight of $V_{\lambda}$ for any $k \in\left\{0,1, \cdots-\left\langle\nu_{1}, \alpha_{i_{0}}\right\rangle\right\}$ and $\nu_{2}-k \alpha_{i_{0}}$ is a weight of $V_{\mu}$ for any $k \in\left\{0,1, \cdots\left\langle\nu_{2}, \alpha_{i_{0}}\right\rangle\right\}$ [cf [sections (13.4) and (21.3) of [8]]]. In particular $\Gamma \nu_{1}+\alpha_{i_{0}}=\lambda$ is a weight of $V_{\lambda}$ (which is ofcourse trivial) and $\nu_{2}-\alpha_{i_{0}}$ is a weight of $V_{\mu}$ and we also have $\nu=\lambda+\nu_{2}-\alpha_{i_{0}}$. Therefore $\Gamma$ in this case $\Gamma$ we take $\lambda_{1}=\lambda$ and $\nu_{2}^{\prime}=\nu_{2}-\alpha_{i_{0}}$.

Now $\Gamma$ let $N$ be a positive integer strictly bigger than 1 . Let us assume that the statement is true for all $\nu$ such that $\nu=\nu_{1}+\nu_{2} \Gamma$ where $\nu_{1}=\lambda-\sum_{i=1}^{l} m_{i} \alpha_{i}$ being a weight of $V_{\lambda}$ with the sum $\sum_{i=1}^{l} m_{i} \leq N-1$ and $\nu_{2}$ being a weight of $V_{\mu}$.

Let $\nu$ be a dominant weight such that $\nu \leq \lambda$ and $\nu=\nu_{1}+\nu_{2}$ where $\nu_{1}$ is a weight of $V_{\lambda} \Gamma \nu_{2}$ is a weight of $V_{\mu}$ with $\nu_{1}=\lambda-\sum_{i} m_{i} \alpha_{i}$ and the $\operatorname{sum} \sum_{i=1}^{l} m_{i}=N$. If $\nu_{1}$ is dominant $\Gamma \operatorname{then} \Gamma$ there is nothing to prove. Otherwise $\Gamma$ there is a simple root $\alpha_{i_{0}}$ such that $\left\langle\nu_{1}, \alpha_{i_{0}}\right\rangle<0$.

By a proof similar to the case when $\sum_{i}^{l} m_{i}=1 \Gamma$ it is easy to see that $\nu_{1}+\alpha_{i_{0}}$ is a weight of $V_{\lambda}$ and $\nu_{2}-\alpha_{i_{0}}$ is a weight of $V_{\mu}$. Also $\Gamma$ we have $\nu=\left(\nu_{1}+\alpha_{i_{0}}\right)+\left(\nu_{2}-\alpha_{i_{0}}\right)$. Therefore $\Gamma$ by induction $\Gamma$ there exist a dominant weight $\lambda_{1} \leq \lambda$ and a weight $\nu_{2}$ of $V_{\mu}$ such that

$$
\nu=\lambda_{1}+\nu_{2}^{\prime}
$$

We fix our choice of $\lambda_{1}$ and $\nu_{2}^{\prime}$ as above. Since $\nu_{2}^{\prime}$ is a weight of $V_{\mu} \Gamma w\left(\nu_{2}^{\prime}\right)$ is also a weight of $V_{\mu}$ for every $w \in W$. Thus $\Gamma$ the unique dominant weight (say $\mu_{1}=w^{-1}\left(\nu_{2}^{\prime}\right)$ ) in the $W$ orbit of $\nu_{2}^{\prime}$ must also be a weight of $V_{\mu}$.

Hence $\Gamma$ we have $\nu=\lambda_{1}+w\left(\mu_{1}\right)$ where $\lambda_{1}$ is a dominant weight satisfying $\lambda_{1} \leq \lambda \Gamma \mu_{1}$ is a dominant weight satisfying $\mu_{1} \leq \mu$ and $w$ is an element of the Weyl group $W$.

Now $\Gamma$ the fact that $V_{\nu}$ is a $\widehat{G}$ - submodule of the tensor product $V_{\lambda_{1}} \otimes V_{\mu_{1}}$ follows from PRV Conjecture [cf [9]]. 
This completes the proof of this Lemma.

We have

Corollary 3.3. Let $\lambda$ and $\mu$ be two dominant weights. Then, the multiplication map

$$
H^{0}\left(X, L_{\lambda}\right) \bigotimes H^{0}\left(X, L_{\mu}\right) \rightarrow H^{0}\left(X, L_{\lambda+\mu}\right) \text { is onto. }
$$

Proof: Proof of this is an immediate consequence of Lemmas 3.1 and 3.2.

Theorem 3.4. Let $G$ be a semisimple adjoint group over the field of complex numbers. Let $\pi: \widehat{G} \longrightarrow G$ be a simply connected covering of $G$. Let $X$ denote the wonderful compactification of $G$. Let $L$ be an ample line bundle over $X$. Consider the embedding of $X$ in $\mathbb{P}\left(H^{0}(X, L)^{*}\right)$. Let $\widehat{X}$ denote the cone over $X$ with respect to this embedding of $X$. Then, $\widehat{X}$ is normal.

Proof: By [4] $\Gamma$ we have $L=L_{\lambda}$ for some regular dominant weight $\lambda$. By [II (5.14)(d)] of [7] $\Gamma$ it suffices to prove that the multiplication map

$$
H^{0}\left(X, L_{\lambda}\right)^{\otimes N} \longrightarrow H^{0}\left(X, L_{N \lambda}\right)
$$

is surjective for every positive integer $N$. The proof of this is an immediate consequence of Corollary 3.3.

We have

Corollary 3.5. Let $w$ be an element of the Weyl group $W$. Let $X_{w}$ denote the scheme theoretic closure of the cell BwB in $X$. Let $L$ be a $\widehat{G} \times \widehat{G}$ linearised very ample line bundle over $X$ and let $\widehat{X_{w}}$ denote the cone over the embedding given by L. Then, $\widehat{X_{w}}$ is normal.

Proof: By [Corollary $3 \Gamma[3]] \Gamma$ the restriction map $H^{0}\left(X, L^{\otimes n}\right) \longrightarrow H^{0}\left(X_{w}, L^{\otimes n}\right)$ is surjective for every positive integer $n \cdots(3.5 .1)$.

Now consider the following commutative diagram of maps:

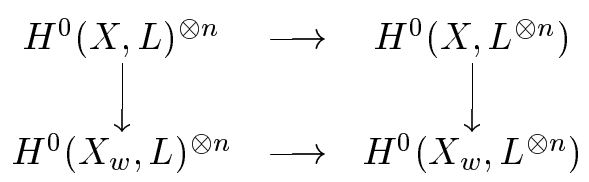

Here the horizontal arrows are the natural multiplication maps and the vertical arrows are restriction maps.

The assertion of the corollary follows from the observations 3.5.1Г3.5.2 and Corollary 3.3. 


\section{Appendix:}

In this section $\Gamma$ we prove the analogue of Theorem 3.4 when the base field is of positive characteristic.

Let $k$ be an algebraically closed field of positive characteristic. Let $G$ be a semisimple adjoint group over $k$. Let $\widehat{G}$ be a simply connected covering of $G$. Let $X$ denote the wonderful compactification of $G$. Let $T \Gamma B \Gamma B^{-} \Gamma \widehat{T} \Gamma \widehat{B} \Gamma \widehat{B^{-}}$be as in section 2 .

With the notations as in section $2 \Gamma$ we introduce some more notations as follows: Let $\chi$ denote the weight lattice of $G$ with respect to $T$. So $\Gamma \chi \times \chi$ is the weight lattice of $G \times G$ with respect to $T \times T$.

For any weight $\lambda \in \chi \Gamma$ set $V_{\lambda}:=H^{0}\left(G / B, L_{w_{0}(-\lambda)}\right)$.

Definition 4.1. Let $\widehat{G}$ be as above. Let $V$ be a $\widehat{G}$ module. A filtration of $\widehat{G}$ - submodules (0) $=V_{0} \subset V_{1} \subset V_{2} \subset \cdots V_{n}=V$ is a good filtration if each $V_{i+1} / V_{i}$ is a direct sum of $V\left(\lambda_{i(j)}\right)$ 's for some dominant weights $\lambda_{i(j)}$ 's.

As in section $2 \Gamma$ let $\Delta=\left\{\alpha_{1}, \cdots \alpha_{l}\right\}$ denote the set of simple roots with respect to $T$ and $B$.

Let $\lambda$ and $\mu$ be two dominant weights in $\chi$.

Let $L_{\lambda}$ (resp. $\left.L_{\mu}\right)$ denote the line bundle over $X$ associated to $(\lambda,-\lambda)$ (resp. to $(\mu,-\mu)$. Let $\nu$ be a dominant weight such that $\nu \leq \lambda+\mu)$. Let $\phi: H^{0}\left(X, L_{\lambda}\right) \otimes H^{0}\left(X, L_{\mu}\right) \rightarrow H^{0}\left(X, L_{\lambda+\mu}\right)$ denote the multiplication map.

ThenГwe have

Lemma 4.2. The $\widehat{G} \times \widehat{G}$ - module $V_{w_{0}(-\nu)} \otimes V_{\nu}$ is a $\widehat{G} \times \widehat{G}$-subquotient of the image $\phi\left(H^{0}\left(X, L_{\lambda}\right) \otimes H^{0}\left(X, L_{\mu}\right)\right)$.

Proof: By Donkin's conjecture [cf Theorem 4.4.3Г[11]] Гa tensor product of two modules with good filtrations has a good filtration. By Theorem 5.10 of $[5] \Gamma H^{0}\left(X, L_{\lambda_{1}}\right)\left(\operatorname{resp} . H^{0}\left(X, L_{\mu_{1}}\right)\right)$ has a good filtration of $\widehat{G} \times \widehat{G}$-submodules such that the successive quotients look like $V_{w_{0}(-\nu)} \otimes V_{\nu} \Gamma$ $\nu \leq \lambda_{1}$ (resp. $\left.\nu \leq \mu_{1}\right)$. Therefore $\Gamma$ the tensor product $H^{0}\left(X, L_{\lambda_{1}}\right) \otimes H^{0}\left(X, L_{\mu_{1}}\right)$ has a good filtration of $\widehat{G} \times \widehat{G}$-submodules.

Now let $H$ be a semisimple Chevalley algebraic group over the field of complex numbers. Let $H_{p}$ be the corresponding algebraic group in characteristic $p$. Now $\Gamma$ if $V_{p}$ is a $H_{p}$-module having a good filtration in characteristic $p$ such that it comes from a $H$-module $V$ modulo $p \Gamma$ 
then the character of $V$ is same as the chracter of $V_{p}$ and so the $H_{p^{-}}$subquotients of $V_{p}$ are precisely the $H$ - direct summands of $V$.

Now $\Gamma$ let $M_{\nu}$ (resp. $M_{\nu}^{\prime}$ ) be the largest $\widehat{G} \times \widehat{G}$ submodule of the tensor product $H^{0}\left(X, L_{\lambda_{1}}\right) \otimes H^{0}\left(X, L_{\mu_{1}}\right)$ all of whose $B \times B$ - weights are $\leq\left(-w_{0}(\nu), \nu\right)\left(\right.$ resp. $\left.<\left(-w_{0}(\nu), \nu\right)\right)$. Also $\Gamma$ let $N_{\nu}$ and $N_{\nu}^{\prime}$ be the corresponding submodules of $H^{0}\left(X, L_{\lambda_{1}+\mu_{1}}\right)$. Therefore $\Gamma$ from

Lemmas 3.1 and $3.2 \Gamma$ and by the observation above $\Gamma \phi$ induces a non zero $\widehat{G} \times \widehat{G}$ - module homomorphism from the quotient $\left(V_{-w_{0}(\nu)} \otimes V_{\nu}\right)^{m}=M_{\nu} / M_{\nu}^{\prime} \longrightarrow N_{\nu} / N_{\nu}^{\prime}=V_{-w_{0}(\nu)} \otimes V_{\nu}$ for some positive integer $m$. Therefore $\Gamma$ by the Frobenius reciprocity [cf Proposition 2.1.6 [11]] $\Gamma$ the assertion follows.

The Theorem (3.4) for positive characteristic follows from Lemma(4.2).

Acknowledgements: We thank Professor C.De Concini for suggesting the problem. We thank Professors M.S.Narasimhan and C.Procesi for their encouragement and useful discussions. We thank ICTP for the hospitality during the stay. We thank the referee for the useful comments and for suggesting the problem for positive characteristics.

\section{References}

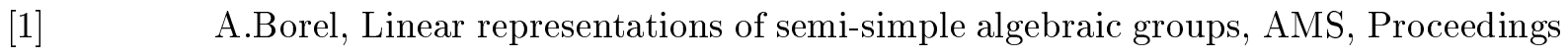
of Symposia in Pure Mathematics $\Gamma$ volume $29 \Gamma 1974$.

[2] N.BourbakiГGroupes et Algebres de LieГCh $4 \Gamma 5$ et 6 ГParis: HermannГ1968.

[3] M.Brion and P.PoloГ Large Schubert Varieties $\Gamma$ Representation Theory 4 (2000) 97-126.

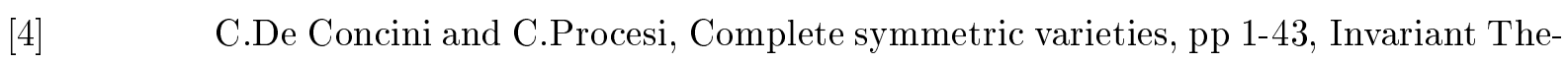
oryГ 996ГLecture Notes in Mathematics.

[5] C.De Concini and T.A.SpringerTCompactification of symmetric varietiesTTransformation GroupsГVol.4ГNo.2-3Г1999Гpp. 273-300.

[6] G.Faltings $\Gamma$ Explicit resolution of local singularities of moduli spaces $\Gamma J$. reine angew. Math. 483 (1997) pp. 183-196.

[7] R.HartshorneГAlgebraic GeometryГSpringer-Verlag $\Gamma 1977$.

[8] J.E.Humphreys Introduction to Lie algebras and representation theory $\Gamma$ Springer Verlag $\Gamma 1972$. 
[9] S.KumarCProof of the Parthasarathy-Ranga Rao-Varadarajan ConjectureГInvent. Math. 93Г117-130 (1988).

[10] E. Strickland $\Gamma$ A Vanishing result for group compactifications $\Gamma$ Math. Ann. 277 (1987)Г165-171.

[11] Wilberd van der KallenГOn Frobenius Splittings and B-ModulesГTIFR Lecture notes in Maths $\Gamma$ Springer-Verlag $\Gamma 1993$. 